\title{
Respiration and Heart Rate Monitoring from Photoplethysmograph Signal
}

\author{
Kiran Balaji PS ${ }^{*}$, Anand Jatti \\ Department of Instrumentation, RV College of Engineering, India
}

Copyright $@ 2015$ Horizon Research Publishing All rights reserved.

\begin{abstract}
Photoplethysmograph is a simple and cost effective method to assess cardiovascular related parameters such as heart rate, arterial blood oxygen saturation, BP the fact that the Photoplethysmograph (PPG) signal caries respiratory information. Earlier physicians used to insert separate sensors to assess respiration and heart rate. In this present work, an efficient algorithm is presented, based on the wavelet decomposition technique to extract the respiratory activity from the PPG signals. With this aim displaying of heart rate and the extraction of respiratory activity is done. Here MATLAB and MULTISIM tools were used for simulation. Wavelet decomposition performed exceptionally well for extraction of respiratory activity from PPG. Extracted signal is compared with a signal which was acquired from the existing respiratory sensor and the correlation between them is up to $90 \%$. Then the obtained parameters are sent to the physician's mobile phone for faster and better assessment of the patient from remote places. Hence using this method it can reduce the usage of sensor for respiration, and also reduces the discomfort caused to the patient, also cost effective.
\end{abstract}

Keywords Heart Rate, Photoplethysmogram (PPG), Respiratory Signal, Wavelets

\section{Introduction}

Respiration monitoring is significant in clinical diagnostics, therapeutics, prognosis, during surgical procedures, post-operative care units, in addition to, monitoring sleep disorders, drug administration and cardiopulmonary disorders. Recording respiratory signal is generally carried out by special instruments and techniques like spirometer, pneumotachometry, capnography, nasal thermistors and whole body plethysmography. This specialized equipment can't be used in all situations and of patient conditions, as the equipment causes either inconvenience or interferes with normal breathing. Therefore methods of extracting respiratory information from more commonly used physiological signals such as electrocardiogram (ECG) and photoplethysmogram (PPG) are always demanded by the physicians, avoiding additional equipment for recording of respiratory activity. Hertzman developed a non-invasive, non-occlusive electro optic method of recording heart related information from variations of the blood volume at a particular test site on the body close to the skin and is called as PPG. A photoplethysmogram is obtained by illuminating a part of the body extremities such as index finger or ear lobe with either infrared or red light and acquiring either the reflected or transmitted light [1]. The PPG signal is composite in nature and has five different frequency components in the interval $0.007-1.5 \mathrm{~Hz}$ [2]. Sources of these frequency components may be relating to respiration, blood pressure control, thermoregulation, autonomous nervous system (ANS) and heart synchronous pulse waveform. Out of the five components, only two components dominate the signal, one is due to the pulsatile component corresponding to the blood flow in the vessels, i.e. the arterial pulse, caused by the heartbeat, and gives an alternating signal (AC component) and the other is, large quasi-DC component that relates to the tissues, bones and to the average blood volume which gives a steady signal. The DC component varies very slowly due to Respiration.

The fundamental frequency of pulsatile AC component is usually around $1 \mathrm{~Hz}$, and varies according to the heart rate of subject under test. The pulsatile AC component is superimposed on to a large value of quasi-DC component. Therefore, the PPG signal not only consists of rhythmic heartbeat information but also a periodic respiratory signal with a frequency band of $0.2-0.33 \mathrm{~Hz}$, due to respiratory induced intensity variation (RIIV) in PPG signal. Hence, the heart rate (or pulse rate) and respiratory rate are two important vital signals, they are of great importance in monitoring health of critically ill adults and infants [3]-[4]. Electrocardiogram (ECG) is used in general by the physicians for clinical monitoring as it represents the electrical manifestation of contractile activity of the heart [5]. It is also evident from the literature that the ECG carries respiratory information and hence much work has been done on ECG derived respiratory activity (EDR) [6]-[7]. In past, researchers were attempting to extract the respiratory 
information embedded in PPG signals in the form of RIIV signal, which includes a simple band pass filter [8], but the band pass filter must be adaptive in nature to allow the band of all possible frequencies corresponding to the respiratory rates. An adaptive band-pass finite impulse response (FIR) filter, designed in frequency sampling method with suitable pass band specifications drawn automatically from the spectrum of the signal itself has efficiently separated heart and respiratory related signals [9]. The proposed method is a robust yet simple method for acquiring the PPG derived respiratory (PDR) signal. Rest of the paper is organised by acquisition circuit for PPG, displaying the heart rate, and extraction of respiratory information from the PPG signal, Results, conclusion.

\section{Methodology of the System}

Initially in this system is powered up using $5 \mathrm{~V}$ power supply. Then the plethesmograph signal is acquired from the index finger using the sensor. The acquired signal is then processed by filtering the DC components and cut off frequency is fixed to avoid the $50 \mathrm{~Hz}$ interference using the LPF and HPF. Then using OpAmp with suitable gain the acquired signal is amplified and then the pulse indicating LED at the output shows the detected PPG signal. Later the amplified signal is transferred to computer using Arduino board where it has an inbuilt ADC. The analog values can be read directly in Arduino software through the serial monitor. Then the displayed values will be saved as a .txt file. Then further the PPG signal is used to detect the heart rate and the respiratory signal is extracted using wavelet decomposition in the Mat-Lab based computer. Suitable GUI is created to access the subject details then choose suitable wavelet, level of decomposition, through the wavelet menu the respiratory activity is displayed. Hence the displayed respiratory rate and heart rate is sent to physician's phone through GSM module. It can be used for the further analysis by the physicians.

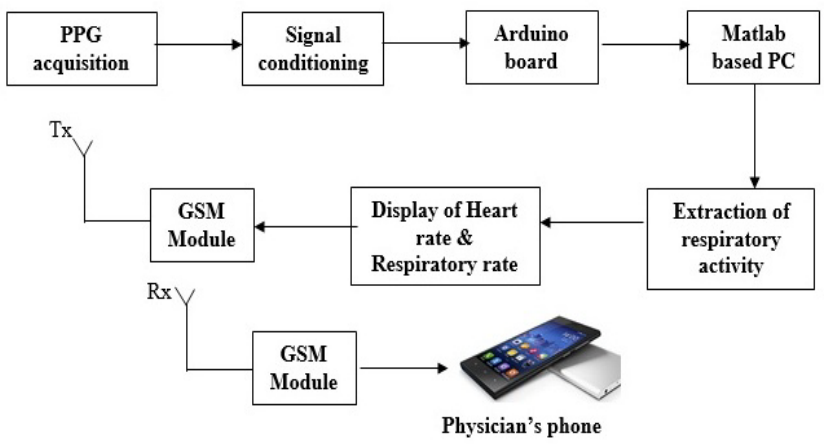

Figure 1. block diagram of overall system

\section{PPG Acquisition System}

In this system the sensor used is TCRT 1000, which is an opto-reflective sensor with IR LED and phototransistor placed adjacently and are bounded inside a leaded package so that there will be least influence of nearby ambient light. The circuit diagram below shows the external biasing circuit for the TCRT1000 sensor. Emitter output of the BJT will turn on the IR LED and trigger the TCRT1000. 220ohms resistor is used to control the current flow and in turn avoids the damage of the IR led. A fingertip positioned over the device, and it will act as a reflecting medium of the light which was incident. The volume of light returned back from the fingertip is supervised by the phototransistor. The reflected light depends on the blood flow in the tissues. In turn blood flow is dependent on heart rate. So the output is synchronous to heart rate.

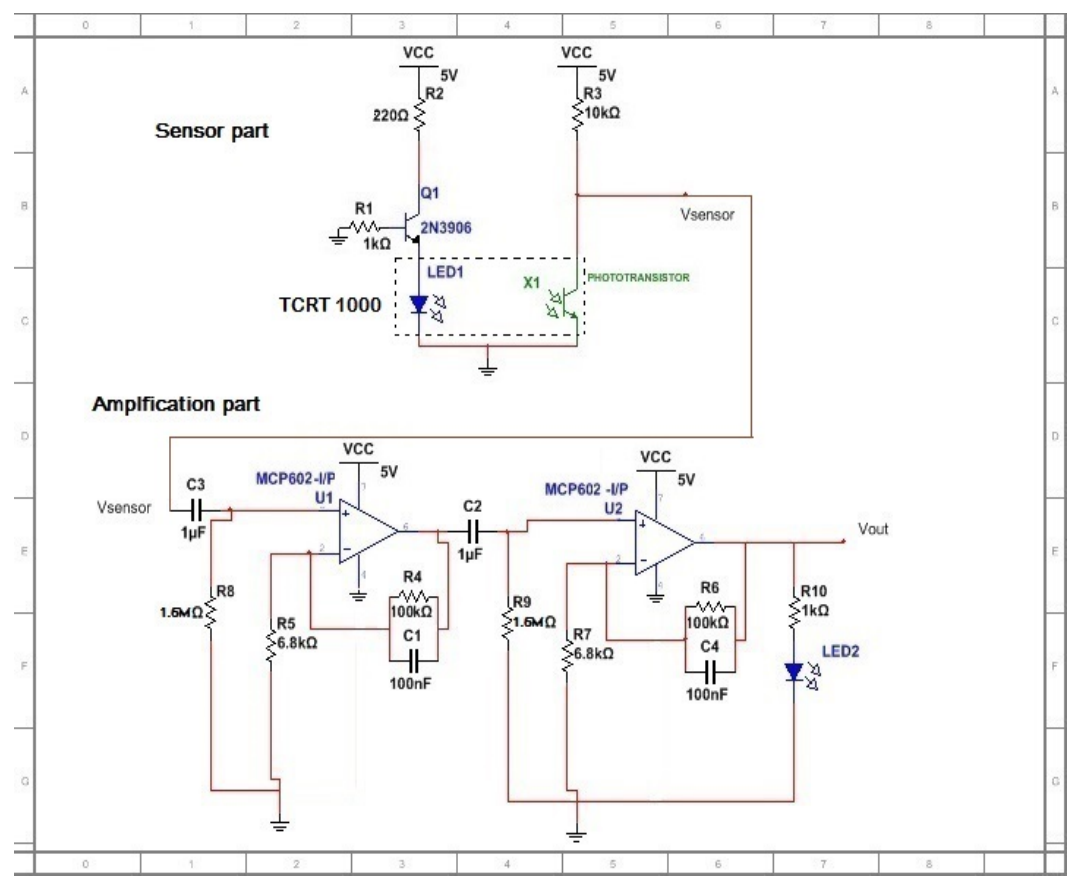

Figure 2. circuit diagram of PPG acquisition unit 
The output (VSENSOR) from the sensor is a periodic physiological waveform attributed to little variations in the reflected IR light which is brought about by the pulsatile tissue blood volume inside the finger. The waveform is, therefore synchronous with the pulse. The circuit diagram depicts the first phase of the signal conditioning which will suppress the expansive DC component and boost the feeble pulsatile $\mathrm{AC}$ part, which carries the required data indicated in Fig 4.1. The sensor output is initially passed through a RC high-pass channel (HPF) to get rid of the DC segment. The cut-off frequency of the HPF is situated to $0.1 \mathrm{~Hz}$. Next stage is active low-pass filter (LPF) that is made of an Op-Amp circuit.

The cut-off frequency of the LPF is set to $15 \mathrm{~Hz}$, respectively. Thus the combination of the HPF and LPF helps to remove unwanted DC signal and high frequency noise including $60 \mathrm{~Hz}(50 \mathrm{~Hz}$ in some countries) mains interference, during the amplification of low amplitude signal (AC component). The output from the first signal conditioning stage goes to a similar HPF/LPF combination for further filtering and amplification (shown in Fig 4.1). The frequency (f) of these pulses is related to the heart rate (BPM) as,

$$
\text { Beats per minute }(\mathrm{BPM})=60 * \text { frequency }
$$

LED which is linked in the 2 stage of the signal conditioning blinks synchronous to the heart beat.

$$
\text { Frequency }=1 / 2 \pi \mathrm{RC} \mathrm{Hz}
$$

$\mathrm{RC}$ - Resistor and Capacitor in LPF and HPF

$$
\begin{gathered}
\text { Gain= } 1+\left(\mathrm{X}_{\mathrm{c}} \| \mathrm{R}_{\mathrm{f}} / \mathrm{R}_{\mathrm{i}}\right) \\
\mathrm{X}_{\mathrm{c}}=\text { Impedance of capacitor } \\
\mathrm{R}_{\mathrm{f}}=\text { Feedback resistor in OpAmp } \\
\mathrm{R}_{\mathrm{i}}=\text { Input resistor in OpAmp }
\end{gathered}
$$

\section{Wavelet Decomposition to Extract Respiratory Activity}

The WT is an interchange to brief time Fourier convert (STFT) or Gabor change. STFT utilizes a solitary examination window, while the WT utilizes variable versatile size window (long window at low frequencies and short window at high frequencies). The WT examination is like time-recurrence investigation focused around WignerVille dispersion. As the biomedical signs are semi occasional in nature and for handling such indicators, wavelet investigation has been ended up being an effective device. Discrete WT (DWT) disintegrates the given indicator into multilevel hierarchic recurrence groups like channel banks. In DWT, a low-pass filter (LPF) and a high-pass filter (HPF) decompose the signal in to different scales. Coefficients of the LPF are referred to as "approximations" defining identity and that of the HPF are referred to as "details", defining imparts of the signal. The approximated signal may be passed down to next stage for further decomposition by breaking the signal content into many levels of lower and components with lower resolution results to a many levels of decomposition. Therefore, the decomposition process is iterative giving rise to wavelet decomposition tree. A block diagram of three level wavelet decomposition tree is shown in Fig.7, where the output approximate coefficients depicted with $A_{j}$ and $D_{j}$ is the detailed coefficients, at that corresponding level results into a set of wavelet sub-band. All detailed coefficients along with approximation coefficients of the last level are used to reconstruct original signal using complementary filters, considering " $\mathrm{M}$ " as the length of the coefficients. ' $\mathrm{L}$ ' is the level of decomposition which results in $\mathrm{L}_{\text {th }}$ approximation of sub band coefficients $\mathrm{cAL}$, and $\mathrm{j}_{\text {th }}$ detailed sub-band coefficients $\mathrm{cD}_{\mathrm{j}}$, where $j=1$, $2, \ldots, L . N$ number of multivariate signals are collected in a matrix to form a wavelet sub-band matrix. Wavelet decomposition can be regarded as projection of the signal on the set of wavelet basis vectors each wavelet coefficient can be computed as the dot product of the signal with the corresponding basis vector. After wavelet decomposition select the suitable detailed coefficients which shows the extracted respiratory signal from the PPG signal. Then compare the signal which is extracted with the breathe signal which was acquired from the existing respiratory sensor. And calculate the correlation between those compared signals to ensure the clinical importance.

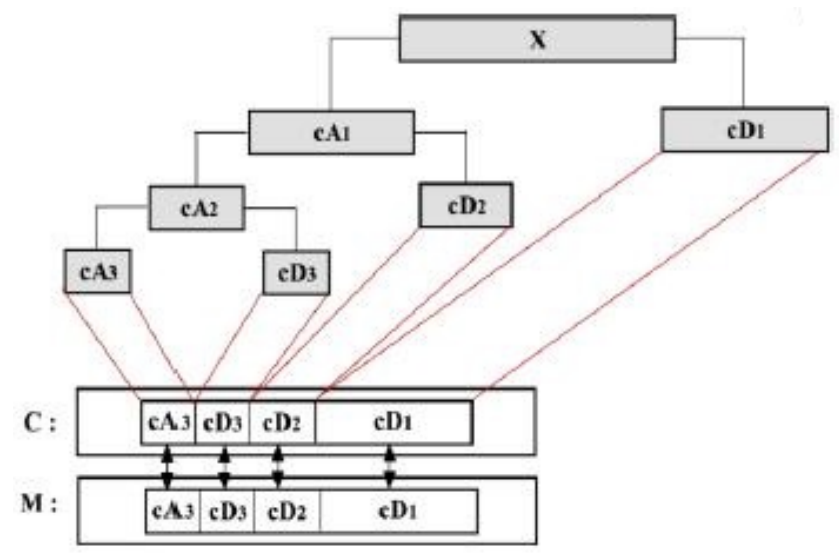

Figure 3. Wavelet Decomposition Tree.

\subsection{Steps for Extraction}

The following are the steps carried out for the respiratory signal extraction using the PPG signal and associated GUI is also displayed in this section

\section{1) Open the wavemenu dialog box}

In the command window use the command as wavemenu to open the wavelet tool box. "Wavedec" performs a multilevel one-dimensional wavelet analysis using either a specific wavelet ('wname') or a specific wavelet decomposition filters (Lo_D and Hi_D). From the dialog box choose 1-D wavelet decomposition for the further processing of the original signal. 


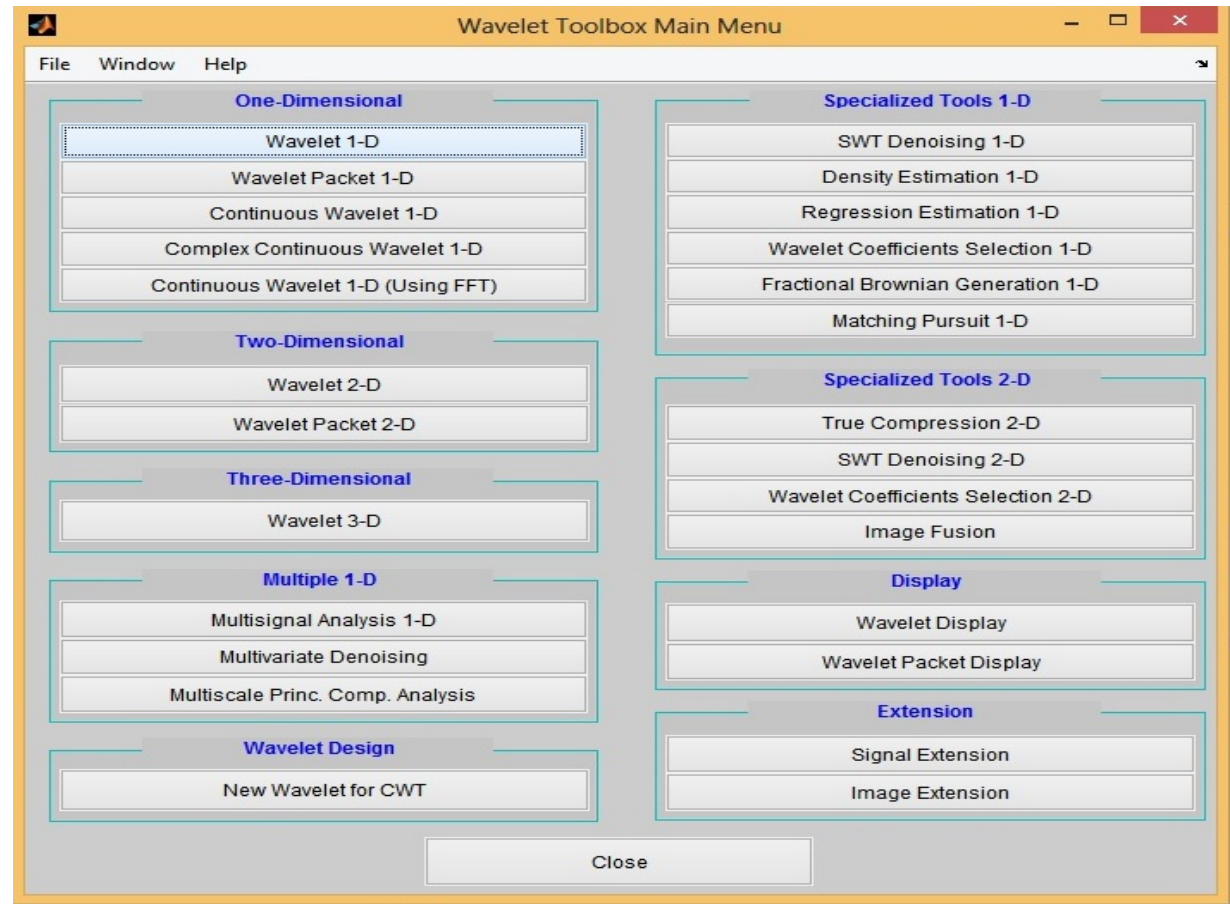

Figure 4. Wavemenu Dialog box

2) Load the PPG signal for extraction

After choosing 1-D wavelet decomposition then Goto file menu choose the respective file which is a .mat file for extraction

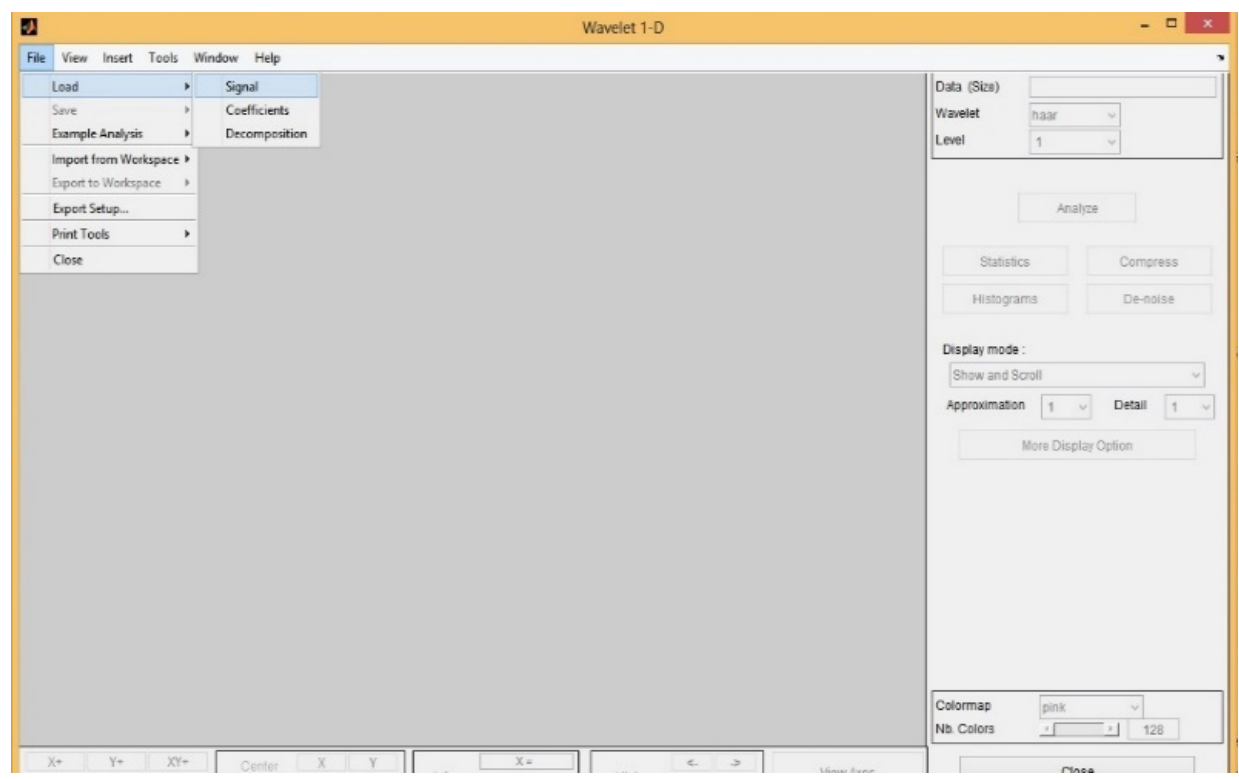

Figure 5. GUI to load the signal

3) Choose suitable wavelet and level of decomposition

After loading the PPG signal then goto wavelet dialog box to choose the suitable wavelet and level of decomposition. here Daubechies wavelet which is a common wavelet for decomposition and level is selected based on the frequency which has to be extracted from the original signal. 


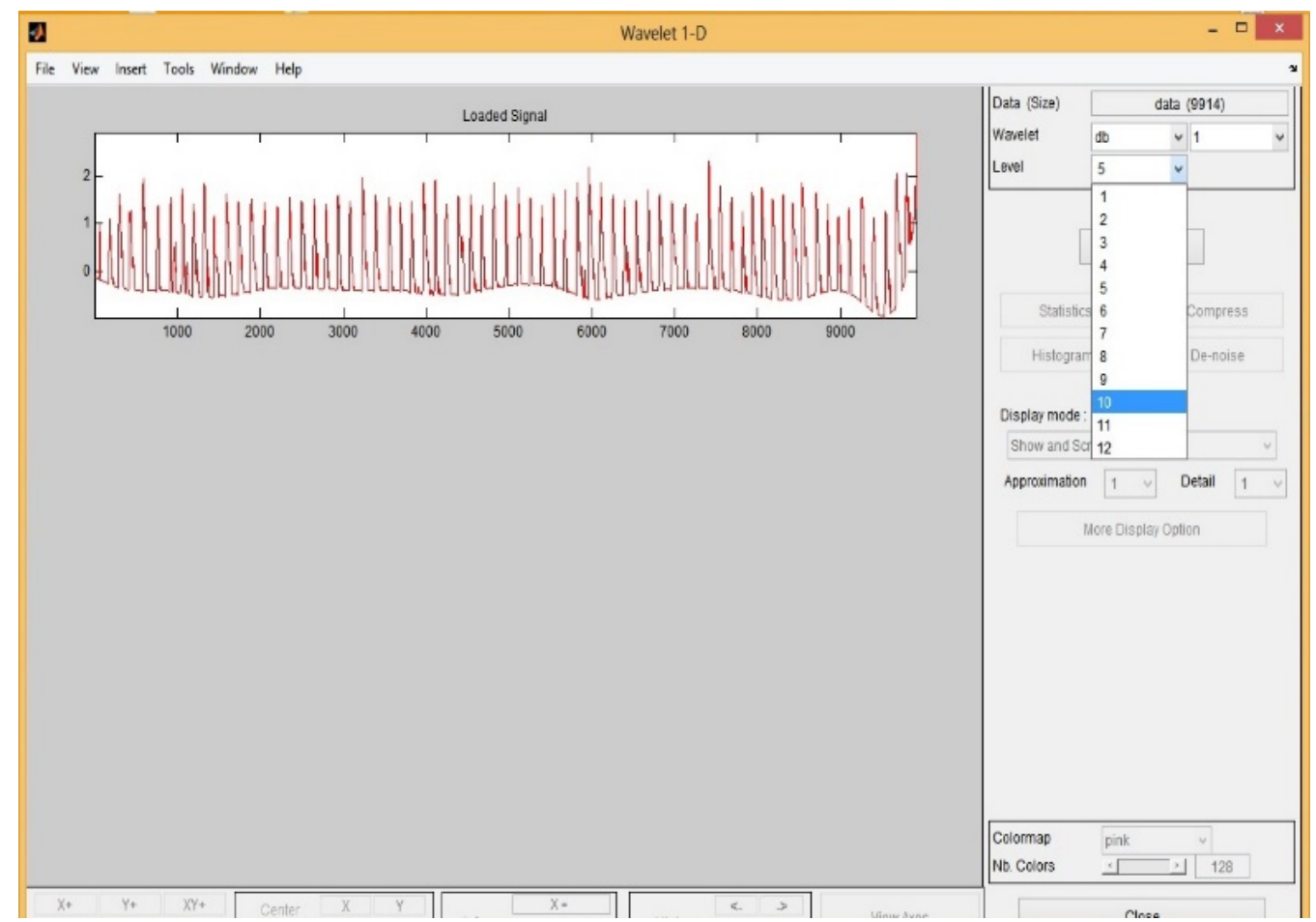

Figure 6. GUI to choose wavelet and level of decomposition

4) Choose the detailed coefficient which indicates the respiratory signal

Here it shows all the wavelet coefficients which were decomposed using wavelet decomposition and using these coefficients the respiratory signal can be obtained. Based on the frequency of data acquisition the D9 coefficient shows the extracted respiratory signal. Then the extracted data is sent to physician's phone through GSM module for further analysis of the patient.

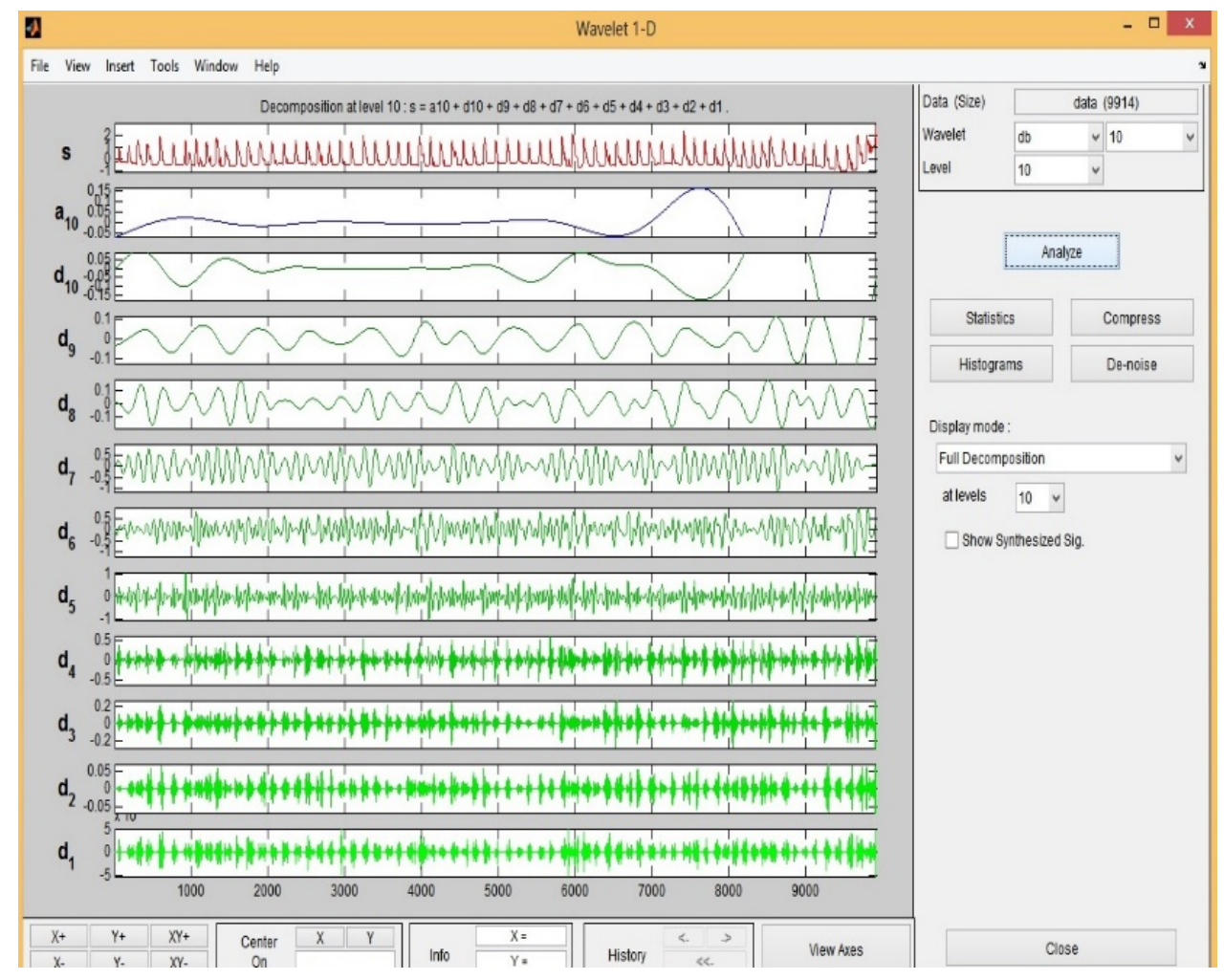

Figure 7. GUI of wavlet decomposed signal with wavelet coefficients 


\section{Experimental Results}

The below displayed image is the snapshot of the GUI which is created in MatLab for accessing the medical data in an efficient and easier way. This is comprised of different subject's data, heart rate of the particular subject data. The acquired PPG signal is also displayed for further analysis. Here from the drop down menu select the subject data as per the requirement. Then click on the heart rate push button for calculating the heart rate of the selected subject. Then use the wavelet toolbox for applying wavelet decomposition. Later the extracted image is compared with the respiratory acquired image for clinical relevance. Then the send to mobile push button is used to send the valuable data to physician's mobile for further analysis.
After doing all the necessary changes in the wavelet toolbox the respiratory data is extracted. The below displayed image is the snapshot of the extracted respiratory signal using the wavelet decomposition by choosing suitable wavelet type, wavelet level. The red coloured signal is the original PPG signal and the green coloured signal is extracted respiratory signal. The rising edge indicates the inspiration and falling edge indicates expiration. The wavelet tree towards the left in the image shows how the wavelet decomposition has occurred and the respective wavelet coefficients. Coefficient " $a$ " is approximation and "d" is detailed. The respiratory signal is obtained from the detailed coefficient.

After the extraction the estimated Respiratory Rate is $=$ 15 breaths/ $\min$

\subsection{Extracted Respiratory Activity in MATLAB}

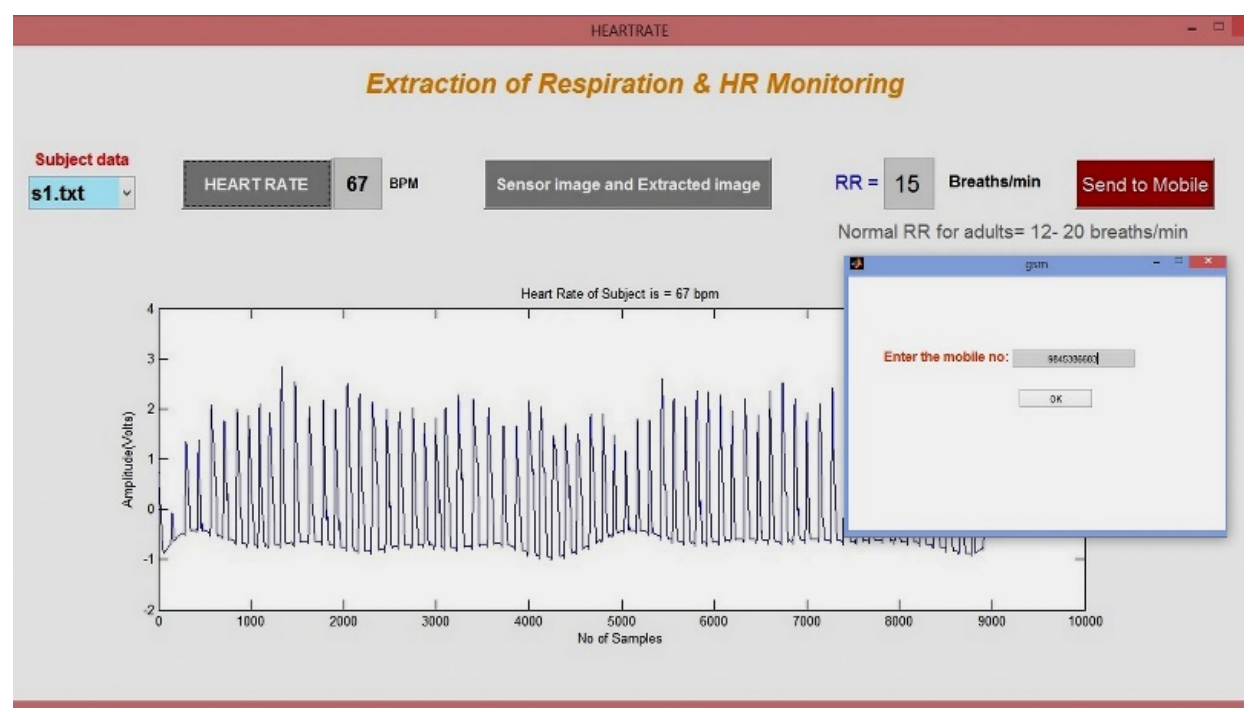

Figure 8. GUI created for accessing medical data
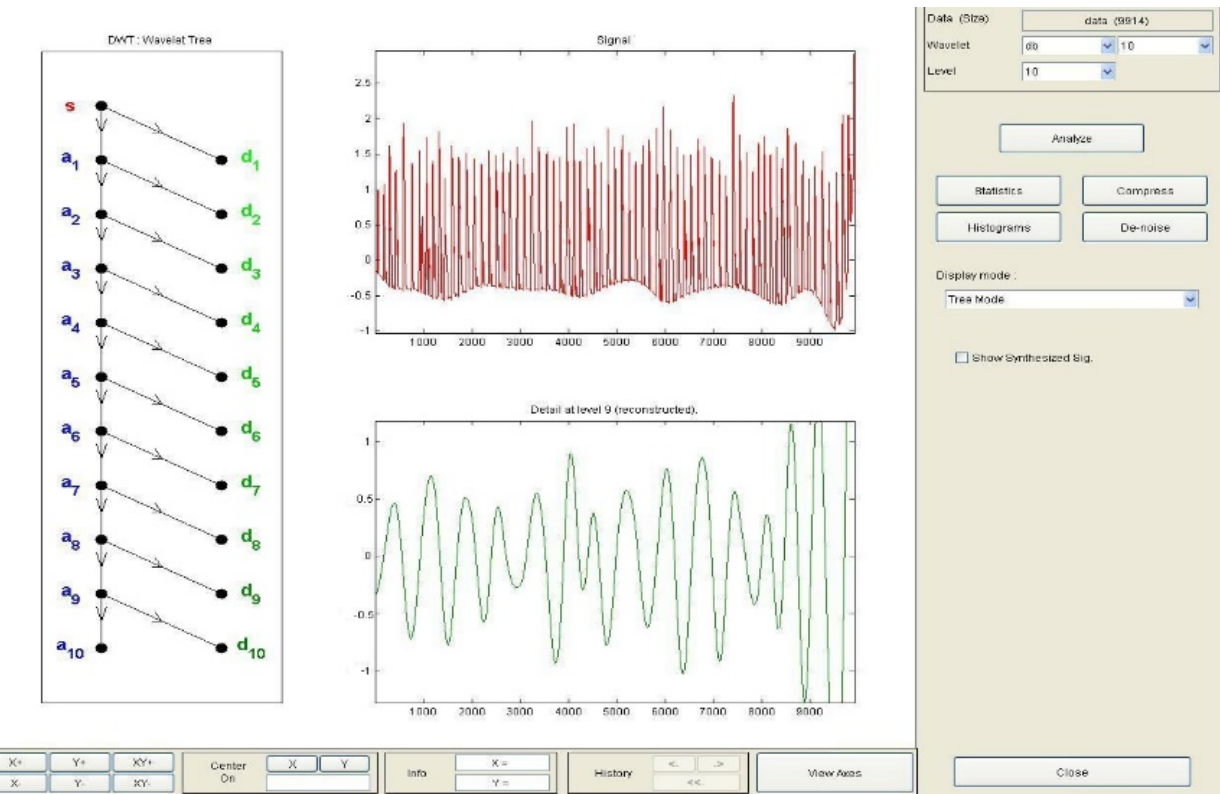

Figure 9. Snapshot of the Extracted respiratory activity from PPG signal for Subject 1 


\subsection{Output in Mobile Phone}

This section is comprised of results of the patient's data which is sent to physician's mobile phone from remote places during an emergency. Below shown fig. 10 is the snapshot of the message which is received in the physician's mobile phone. It displays the parameters like heart rate and respiratory rate of the particular patient.

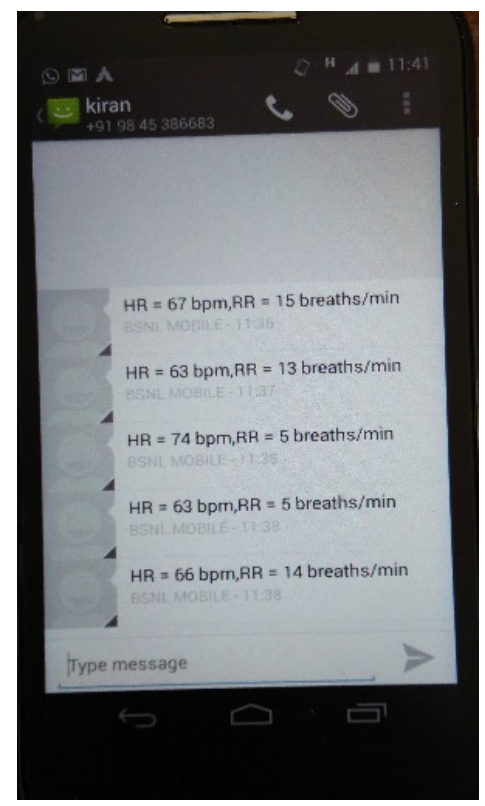

Figure 10. Patient details sent to physician's mobile phone

\section{Discussion on the Results}

Below shown fig.11 shows that there is a strong correlation between the extracted signal and acquired signal from currently used respiratory sensors.

Table 1 shows the correlation results between the extracted signal and the acquired signal which implies the proposed method can extract the respiratory signal from the PPG signal and it has the correlation up to $90 \%$.

Table 1. correlation between extracted signal and acquired signal

\begin{tabular}{|c|c|c|c|}
\hline $\begin{array}{c}\text { Subject } \\
\text { No }\end{array}$ & $\begin{array}{c}\text { Acquired } \\
\text { Respiratory Rate }\end{array}$ & $\begin{array}{c}\text { Extracted } \\
\text { Respiratory Rate }\end{array}$ & $\begin{array}{c}\text { Correlation } \\
\text { (In \%) }\end{array}$ \\
\hline 1 & 16 & 15 & 93.75 \\
\hline 2 & 12 & 13 & 92.3 \\
\hline 3 & 6 & 5 & 83.33 \\
\hline 4 & 7 & 5 & 71.4 \\
\hline 5 & 15 & 14 & 93.33 \\
\hline 6 & 17 & 13 & 76.4 \\
\hline 7 & 14 & 12 & 85.7 \\
\hline 8 & 5 & 4 & 80 \\
\hline 9 & 6 & 5 & 83.33 \\
\hline 10 & 15 & 13 & 86.66 \\
\hline 11 & 18 & 16 & 88.88 \\
\hline 12 & 16 & 15 & 93.75 \\
\hline 13 & 9 & 10 & 90 \\
\hline 14 & 8 & 9 & 88.88 \\
\hline 15 & 13 & 14 & 92.85 \\
\hline
\end{tabular}

(a)

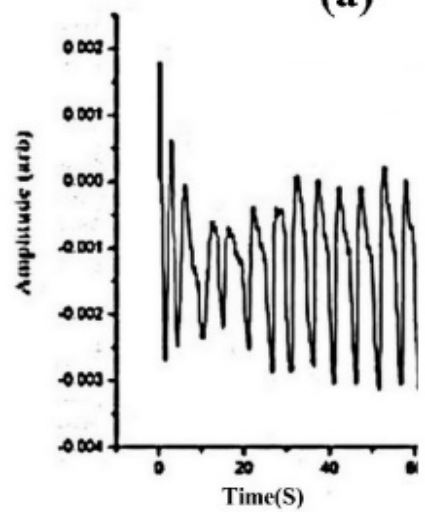

(b)

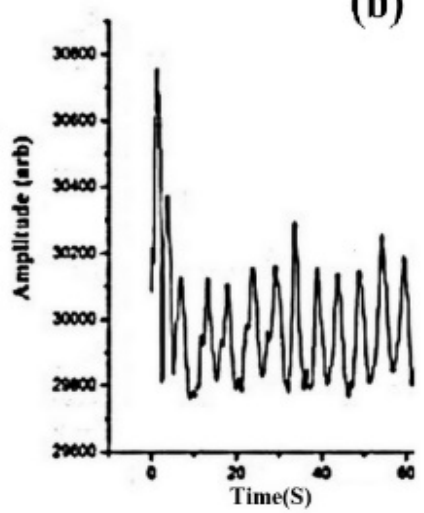

(c)

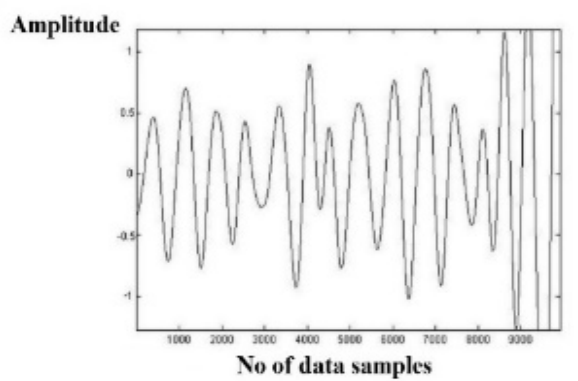

Figure 11. Comparison with respiratory sensor (a) Respiratory signal from thoracic belt (b) Fibre optic sensor (c) Extracted signal from PPG signal 


\section{Comparison of extracted and acquired respiratory rate}

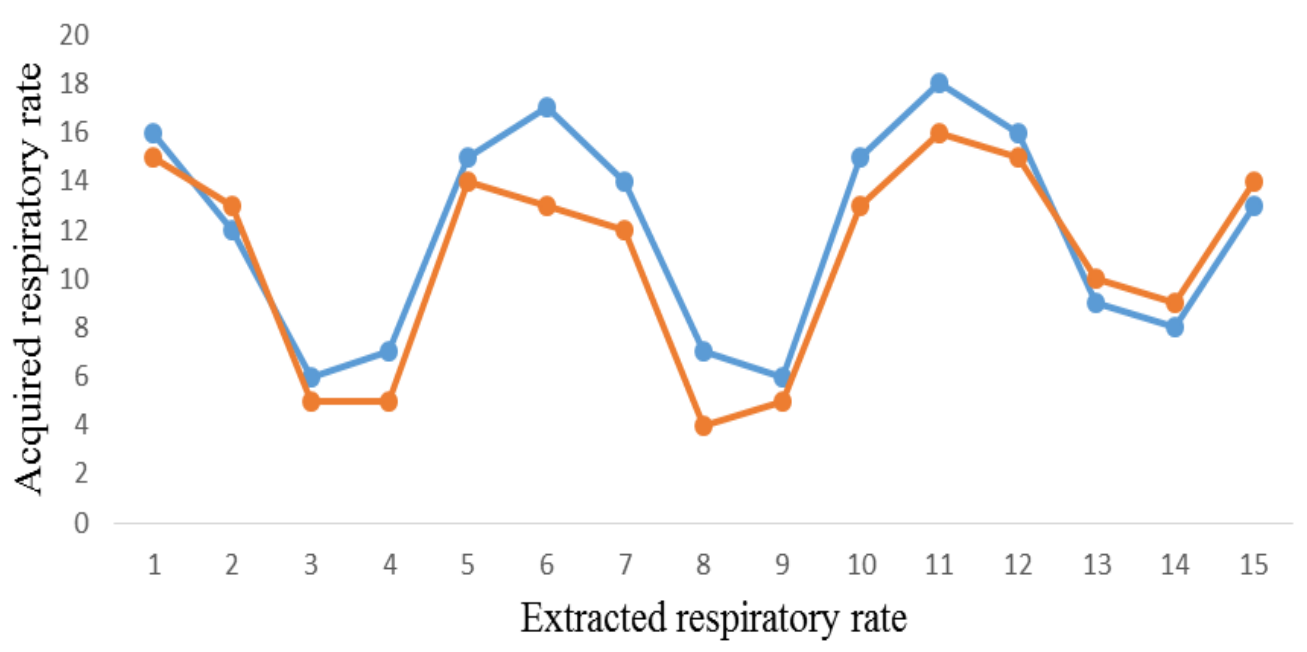

- Acquired Respiratory Rate $\quad \longrightarrow$ Extracted Respiratory Rate

Figure 12. Graph which shows the correlation between extracted data and acquired data

\section{Conclusions}

A simple prototype of the hardware has been implemented suitable GUI has been created in MATLAB for easy accessing and analysis. So an efficient method has been used for extracting respiratory data from the PPG signal which is very much necessary and challenging. In turn this helps in avoiding the use of another sensor, which will cause discomfort for the subject and also the cost of the respiratory sensor can also be reduced. The extraction of respiratory signal is carried out by employing wavelet decomposition method using Daubechies wavelets. This has been a very promising technique for extraction of respiratory data from physiological signs which is having incredible breathing impact. The correlation between extracted signal and acquired signal is more than $90 \%$. This system also helps to send the valuable patient's data to physician's mobile during an emergency situation which is an added advantage.

This system can be enhanced further by using some better extraction techniques to improve the precision of the extracted signal and to improve the clinical importance of the signal. Instead of text message if it can be made to send the image of the acquired and extracted data it will be more useful for physicians for better understanding of the patient's condition and take suitable quick action.

\section{REFERENCES}

[1] J. G. Webster, Medical Instrumentation: Application and Design, 4th Ed. New York: Wiley, 2010.

[2] J. B. West, Respiratory Physiology: The essentials. Philadelphia, PA: Lippincott Williams \& Wilkins, 2008.
[3] A. B. Hertzman, "The blood supply of various skin areas as estimated by photoelectric plethysmograph," Amer. J. Physiol., vol. 124, no. 2, pp. 328-340, Oct. 1938.

[4] J. G. Webster, Design of Pulse Oximeters. New York: Taylor \& Francis, 1997.

[5] A. Johansson, L. Nilsson, S. Kalman, and P. Å. Öberg, "Respiratory monitoring using photoplethysmography," in Proc. 20th Annu. Conf. IEEE EMBS, 1998, vol. 20, no. 6, p. 3226.

[6] J. Li, J. Jin, X. Chen, W. Sun, and P. Guo, "Comparison of respiratoryinduced variations in photoplethysmographic signals," Physiol. Meas., vol. 31, no. 3, pp. 415-425, Mar. 2010 .

[7] W. Einthoven, G. Fahr, and A. Waart, "On the direction and manifest size of the variations of potential in the human heart and on the influence of the position of the heart on the form of the electrocardiogram," Amer. Heart J., vol. 40, no. 2, pp. 163-211, Aug. 1950.

[8] R. Bailon, L. Sornmo, and P. Laguna, "A robust method for ECG-based estimation of the respiratory frequency during stress testing," IEEE Trans. Biomed. Eng., vol. 53, no. 7, pp. 1273-1285, Jul. 2006.

[9] K. Nakajima, T. Tamura, T. Ohta, and H. Miike, "Monitoring of heart and respiratory rates by photoplethysmography using a digital filtering technique," Med. Eng. Phys., vol. 18, no. 5, pp. 365-372, Jul. 1996.

[10] P. A. Leonard, J. G. Douglas, N. R. Grubb, D. Clifton, P. S. Addison, and J. N. Watson, "A fully automated algorithm for the determination of respiratory rate from the photoplethysmogram," J. Clin. Monit. Comput., vol. 20, no. 1, pp. 33-36, Feb. 2006.

[11] L. Nilsson, A. Johansson, and S. Kalman, "Monitoring of respiratory rate in postoperative care using a new photoplethysmographic technique," J. Clin. Monit., vol. 16, no. 4 , pp. 309-315, 2000. 
[12] J. Lee, J. P. Florian, and K. H. Chon, "Respiratory rate extraction from pulse oximeter and electrocardiographic recordings," Physiol. Meas., vol. 32, no. 11, pp. 1763-1773, Nov. 2011.

[13] J. Y. A. Foo and S. J. Wilson, "Estimation of breathing interval from the photoplethysmographic signals in children," Physiol. Meas., vol. 26, no. 6, pp. 1049-1058, Dec. 2005.

[14] Y. D. Lin, W. T. Liu, C. C. Tsai, and W. H. Chen, "Coherence analysis between respiration and PPG Signal by bivariate AR Model," World Acad. Sci. Eng. Technol., vol. 53, pp. 847$851,2009$.

[15] K. H. Chon, S. Dash, and K. Ju, "Estimation of respiratory rate from photoplethysmogram data using time-frequency spectral estimation," IEEE Trans. Biomed. Eng., vol. 56, no. 8, pp. 2054-2063, Aug. 2009.

[16] S. Dash, K. H. Shelley, D. G. Silverman, and K. H. Chon, "Estimation of respiratory rate from ECG, photoplethysmogram, and piezoelectric pulse transducer signals: A comparative study of time-frequency methods," IEEE Trans. Biomed. Eng., vol. 57, no. 5, pp. 1099-1107, May 2010.

[17] K. V. Madhav, M. R. Ram, E. H. Krishna, and K. A. Reddy, "Monitoring respiratory activity using PPG signals by order reduced-modified covariance AR technique," in Proc. 4th IEEE iCBBE, Chengudu, China, Jun. 18-20, 2010, pp. 1-4.

[18] J. Lee and K. H. Chon, “An autoregressive model-based particle filtering algorithms for extraction of respiratory rates as high as 90 breaths per minute from pulse oximeter," IEEE Trans. Biomed. Eng., vol. 57, no. 9, pp. 2158-2167, Sep. 2010.

[19] J. Lee and K. H. Chon, "Time-varying autoregressive model based multiple modes particle filtering algorithm for respiratory rate extraction from pulse oximeter," IEEE Trans. Biomed. Eng., vol. 58, no. 3, pp. 790-794, Mar. 2011.

[20] K. V. Madhav, M. R. Ram, E. H. Krishna, K. A. Reddy, and K. N. Reddy, "Estimation of respiration rate from principle components of photoplethysmographic signals," in Proc. of 2010 IEEE EMBS conf. on Biomed. Eng. \& Sciences, IECBES-2010, Kuala Lumpur, Malaysia, Nov./Dec. 2010, pp. 311-314.

[21] K. V. Madhav, M. R. Ram, E. H. Krishna, K. N. Reddy, and K. A. Reddy, "Estimation of respiratory rate from ECG, BP and PPG signals using empirical mode decomposition," in Proc. 28th IEEE I2MTC, Hangzhou, China, May 10-12, 2011, pp. 1611-1664.

[22] K. V. Madhav, M. R. Ram, E. H. Krishna, K. N. Reddy, and K. A. Reddy, "Use of multi scale PCA for extraction of respiratory activity from photoplethy -smographic signals," in Proc. 29th IEEE I2MTC, Graz, Austria, May 13-16, 2012, pp. 1784-1787.

[23] K. A. Reddy, B. George, and V. J. Kumar, "Use of Fourier series analysis for motion reduction and data compression of photoplethysmographic 